\title{
Kritische postoperative Blutzuckerwerte Der schmale Grat zwischen zu hoch und zu niedrig
}

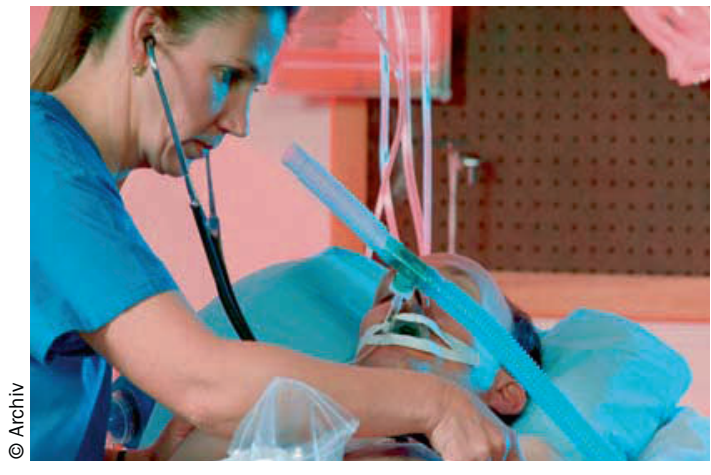

Ist der Blutzucker nach kardiovaskulären Operationen zu hoch oder öfters zu tief, steigen die Komplikationsrate und die Verweildauer auf der Intensivstation.

Fragestellung: Welche Blutzucker-(BZ-)werte sollten bei Patienten mit und ohne Diabetes nach kardiovaskulären Operationen angestrebt, welche vermieden werden, um Komplikationen zu verhindern?

Hintergrund: Diabetiker haben ein erhöhtes postoperatives Risiko nach aortokoronarer Bypassoperation. Doch die Gefahr droht nicht nur von der Stoffwechselerkrankung als solcher, sondern auch von der Höhe der postoperativen Blutzuckerwerte bei Diabetikern und auch bei Nichtdiabetikern.

Patienten und Methodik: Bei 642 Patienten (187 Diabetiker und 455 Nichtdiabetiker, mittleres Alter 68 Jahre) erfolgten 427 Bypass-Operationen allein oder mit anderen Eingriffen kombiniert $(n=122)$, 155 Karotisendarterektomien und 58 vaskuläre Eingriffe an den unteren Extremitäten. Die Patienten beider Gruppen erhielten postoperativ gleich häufig (97 bzw. 98\%) Insulininfusionen.
Ergebnisse: Die Mortalität während des Krankenhausaufenthaltes lag bei Diabetikern mit 5\% höher als bei Nichtdiabetikern mit $2 \%$. Die postoperativen Komplikationen im Gesamtkollektiv stiegen mit der Höhe des Blutzuckers am ersten postoperativen Tag von der untersten zur obersten Quartile an, nämlich von 0 über $1,6 \%$ und $1,7 \%$ bis zu $9 \%$. Bei den Patienten mit BZ-Werten in der obersten Quartile (> 8,8 mmol/l bzw. $160 \mathrm{mg} / \mathrm{dl}$ ) waren Mortalität, Infektionsrate und Verweildauer in der Intensivstation jeweils signifikant erhöht (Tab. 1). Diese Korrelation bestand für die postoperativen BZWerte und war nicht auf Patienten mit präoperativer Diabetesdiagnose beschränkt. Allerdings war auch zu niedriger BZ mit einer schlechten Prognose verknüpft. Denn Patienten mit drei oder mehr Hypoglykämien < 4,1 mmol/l bzw. $75 \mathrm{mg} / \mathrm{dl}$ hatten signifikant höhere Mortalitäts- und Infektionsraten und waren länger auf der Intensivstation.

Schlussfolgerung: Erhöhte Blutzuckerwerte über $8,8 \mathrm{mmol} / \mathrm{l}$ und Hypoglykämien < 4,1 mmol/1 postoperativ nach kardiovaskulären Operationen gehen - mit und ohne Diabetes - mit einer erhöhten Mortalität und Morbidität einher (s.a. ab S. 34).

\begin{tabular}{|c|c|c|c|}
\hline \multicolumn{3}{|c|}{ Tab. 1: Risikosteigerung durch hohen oder niedrigen Blutzucker } \\
\hline Blutzucker & Todesfälle & $\begin{array}{c}\text { Risiko für } \\
\text { Infektionen }\end{array}$ & $\begin{array}{c}\text { Verweildauer > 27 h } \\
\text { auf der Intensivstation }\end{array}$ \\
\hline$>8,8 \mathrm{mmol} / \mathrm{I}$ & 10,16 & 1,76 & 3,10 \\
\hline $4,1 \mathrm{mmol} / \mathrm{I}$ & 9,08 & 8,57 & 4,28 \\
\hline
\end{tabular}

Risikoerhöhung (Hazard Ratio) bei postoperativen Blutzuckerwerten über 8,8 mmol/I bzw. drei und mehr Hypoglykämien unter 4,1 mmol/I bei Diabetikern und Nichtdiabetikern (Adjust. für Alter, Geschlecht, Diabetesdiagnose prä-OP).
Frioud A, ComtePerret S, Nguyen S et al. Blood glucose level on postoperative day 1 is predictive of adverse outcomes after cardiovascular surgery. Diabetes Metab 2010;36(1):36-42.
Kommentar: Die Höhe der Blutzuckerwerte ist ein unabhängiger Prädiktor für Komplikationen nach kardiovaskulären Operationen. Das gilt gleichermaßen für erhöhte und zu niedrige Werte bei Diabetikern und Nichtdiabetikern. Die postoperative Überwachung und optimale Einstellung der Blutzuckerwerte ist eine äußerst schwierige und kritische Aufgabe, weil alle Versuche einer strengen Blutzuckerkontrolle auch die Gefahr einer ebenfalls fol- genschweren Hypoglykämie beinhalten. Leider haben sich Experten und Fachgesellschaften mangels aussagekräftiger bzw. reproduzierbarer Studien noch nicht auf optimale Zielwerte einigen können.

Prof. Dr. med. Heinrich Holzgreve

Internist, Kardiologische Praxis

Burgstr. 7

D-80331 München

h.holzgreve@t-online.de 